\title{
The impact of chronic stress and eating types on active ghrelin levels in response to acute stress
}

\author{
Christine Fahrngruber, Kalina Duszka and Jürgen König \\ Department of Nutritional Sciences, University of Vienna, Vienna, Austria
}

\begin{abstract}
Chronic stress is associated with impacting eating behavior, namely food choice and energy intake, with a shift towards more palatable and energy dense foods. Additionally, eating behavior is influenced by other psychological factors like mood and emotions. The categorization of people into eating types such as restrained, emotional, and external eaters has gained attraction. Reported changes in eating behavior due to psychological stress are only occasionally accompanied by measures of physiological hunger through ghrelin. The primary objective of this study was to investigate how chronic stress and acute cortisol reactivity affect active ghrelin secretion and how these outcomes account for different eating types. 16 healthy, young males (age: $23 \pm 3$ years, BMI: $22.5 \pm 1.3 \mathrm{~kg} / \mathrm{m}^{2}$ ) with low (n $=8)$ and average-to-high $(\mathrm{n}=8)$ chronic stress level were subjected to the Trier Social Stress Test $(\mathrm{TSST})$ and a control version on two separate days. Active ghrelin, cortisol, glucose, and heart rate were measured throughout the test. Subjects rated their hunger by means of visual analog scale and current mood was assessed with the Positive and Negative Affect Scale (PANAS). In addition, participants filled out the Dutch Eating Behavior Questionnaire (DEBQ) to account for their subjective eating behavior. Overall ghrelin values where higher on the test day compared to the control day. Ghrelin values were also higher during the time leading up to the stress or control test (TSST) than during the conclusion of said tests. On both days, mean values for active ghrelin where higher in individuals with low chronic stress exposure compare to those with average-to-high chronic stress exposure. While values from test to control day decreased for lower stressed participants, they slightly increased for higher stressed participants. Cortisol responders displayed higher ghrelin values on test day than cortisol non-responders, but this association inverted for the control day. Results indicate that chronic stress influences acute stress response and further alters active ghrelin production, which in turn can influence eating behavior. Replication in a greater group of participants of differing weight and sex could yield a greater understanding of stress induced eating. Factors such as relaxation techniques and coping mechanisms could further improve our knowledge and evaluate treatment possibilities.
\end{abstract}

\section{Conflict of Interest}

There is no conflict of interest. 